\title{
What explains the relationship between spatial and mathematical skills? A review of evidence from brain and behavior
}

\author{
Zachary Hawes $^{1} \cdot$ Daniel Ansari $^{1}$ \\ Published online: 21 January 2020 \\ (C) The Psychonomic Society, Inc. 2020
}

\begin{abstract}
There is an emerging consensus that spatial thinking plays a fundamental role in how people conceive, express, and perform mathematics. However, the underlying nature of this relationship remains elusive. Questions remain as to how, why, and under what conditions spatial skills and mathematics are linked. This review paper addresses this gap. Through a review and synthesis of research in psychology, neuroscience, and education, we examine plausible mechanistic accounts for the oft-reported close, and potentially causal, relations between spatial and mathematical thought. More specifically, this review targets candidate mechanisms that link spatial visualization skills and basic numerical competencies. The four explanatory accounts we describe and critique include the: (1) Spatial representation of numbers account, (2) shared neural processing account, (3) spatial modelling account, and (4) working memory account. We propose that these mechanisms do not operate in isolation from one another, but in concert with one another to give rise to spatial-numerical associations. Moving from the theoretical to the practical, we end our review by considering the extent to which spatial visualization abilities are malleable and transferrable to numerical reasoning. Ultimately, this paper aims to provide a more coherent and mechanistic account of spatial-numerical relations in the hope that this information may (1) afford new insights into the uniquely human ability to learn, perform, and invent abstract mathematics, and (2) on a more practical level, prove useful in the assessment and design of effective mathematics curricula and intervention moving forward.
\end{abstract}

Keywords Spatial skills $\cdot$ Numerical skills $\cdot$ Spatial visualization $\cdot$ Mathematical cognition

\section{Introduction}

The mapping of numbers to space is central to how we operationalize, learn, and do mathematics. From a historical perspective, it is difficult, if not impossible, to sift through the major discoveries in mathematics without acknowledging the central importance placed on the mapping of numbers to space (Lakoff \& Núñez, 2000). For example, the Pythagorean Theorem, the Cartesian coordinate system (mapping in general), triangular numbers, the real number line, and Cavalieri's principle are but a few famous examples of numerical-spatial mappings (Davis, 2015; Dehaene, 2011; Giaquinto, 2008;

Zachary Hawes

zhawes@gmail.com

1 Department of Psychology, Brain and Mind Institute, University of Western Ontario, Western Interdisciplinary Research Building, Pod A-10, 1151 Richmond Street North, London, ON N6A 5B7, Canada
Hubbard, Piazza, Pinel, \& Dehaene, 2009). More ubiquitous examples include the measurement of time and various other everyday quantities (e.g., cooking ingredients; Newcombe, Levine, \& Mix, 2015). Mathematical instruments as well as measurement devices are in themselves a testament to the widespread application of mapping numbers to space. These examples include the abacus, number line, clock, and ruler. To flip through any mathematical textbook is to further reveal the intimate relations between numbers and space. Diagrams, graphs, and various other visual-spatial illustrations fill the pages and serve to communicate and improve mathematical understanding.

From these examples, it is clear that numbers and space interact in important ways. But how is it that these spatialnumerical associations come to be in the first place? What are the cognitive processes that underlie our uniquely human ability to derive the Pythagorean Theorem or to invent concepts and tools to measure the world around us? In this paper, we ask what role spatial abilities might play in mathematical reasoning. More specifically, we focus on the ways in which 
spatial visualization might facilitate numerical reasoning skills, including the competencies related to basic number sense and operations. Our primary intent is to go beyond the question of whether spatial visualization and numerical abilities are linked and instead examine why they may be linked. The following quote not only speaks to this need, but also makes it clear why we should care about this area of study:

\section{The relation between spatial ability and mathematics is so well established that it no longer makes sense to ask wheth- er they are related. Rather, we need to know why the two are connected-the causal mechanisms and shared processes-for this relation to be fully leveraged by educa- tors and clinicians (Mix \& Cheng, 2012, p. 206).}

To address this gap in the literature, the following review presents four mechanistic accounts of why spatial visualization may be fundamentally linked to numerical reasoning. These four accounts include the: (1) Spatial representation of numbers account, (2) shared neural processing account, (3) spatial modeling account, and (4) working memory account. These accounts are based on a synthesis of literature spanning psychology, neuroscience, and education. They are not mutually exclusive. For example, there is considerable overlap between the spatial representation of numbers account and the shared neural processing account. The extent to which these various accounts are descriptions of the same underlying mechanism but vary according to discipline-specific epistemologies and research traditions, as well as different levels of analyses (behavioral vs. neural), is an important possibility to consider and one that we address in our General discussion. However, for ease of communication and in an attempt to best represent the research traditions in which these accounts originate from, we present them as separate mechanisms. In the end, it is our aim to provide insight and stimulate further questions as to when, why, and how spatial visualization and numerical abilities are linked.

We intentionally target spatial visualization skills in this review because this type of spatial thinking appears to be especially related to mathematical thinking (Mix \& Cheng, 2012; Hawes, Moss, Caswell, Seo, \& Ansari, 2019). For example, while there is little evidence (to date) to suggest that spatial navigation skills relate to mathematics abilities, there is well over a century of research linking spatial visualization and mathematics (Davis, 215; Galton, 1880; Mix \& Cheng, 2012). Broadly defined here as the ability to generate, recall, maintain, and manipulate visual-spatial images and solutions (Lohman, 1996; see Fig. 1), spatial visualization has been reported to play a critical role in mathematical and scientific discovery and innovation. For example, the discovery of the structure of DNA, the Theory of Relativity, the Periodic Table, and the invention of the induction motor are all said to have been borne out of spatial visualization (Davis, 2015; Moss, Bruce, Caswell, Flynn, \& Hawes, 2016; Newcombe, 2010). According to famed mathematician Jacques Hadamard (1945), mathematical discoveries first present themselves in the form of intuitions and visual-spatial imagery. Only then does one engage in the more arduous and time-consuming work of testing the veracity of one's imaginings through formal and symbolic logic. This theory is perhaps best articulated by Albert Einstein, who in a letter to Hadamard, wrote:

The words or language, as they are written or spoken, do not seem to play any role in my mechanism of thought. The physical entities which seem to serve as elements in thought are certain signs and more or less clear images which can be "voluntarily" reproduced and combined. ...Conventional words or other signs have to be sought for laboriously only in a secondary stage, when the mentioned associative play is sufficiently established and can be reproduced at will (Einstein, quoted in Hadamard, 1945, p. 142-143).

Critically, Einstein is not alone in describing his thought process in this way. Many other mathematicians and scientists, including Poincaré, van't Hoff, and Pasteur, have offered similar introspective accounts (Hadamard, 1945; Root-Bernstein, 1985). These anecdotal accounts provide important, but far from conclusive, accounts of the role(s) that spatial visualization might play in mathematical discovery. But what does the empirical evidence suggest? Further, and more to the point, what role do spatial visualization skills play in the learning and performance of school-based mathematics?

In terms of mathematical and scientific discovery and innovations, there is longitudinal support for strong predictive relations (Wai, Lubinski, \& Benbow, 2009). For example, in a nationally representative sample of US high school students $(\mathrm{N}=400,000)$, it was found that spatial visualization abilities predicted which students enjoyed, entered, and succeeded in STEM disciplines (science, technology, engineering, and mathematics), even after taking verbal and quantitative competencies into account (Wai, Lubinksi, \& Benbow, 2009). Follow-up studies of this same sample further demonstrated that spatial visualization skills predicted creativity and innovation in the workplace, suggesting that there may be some truth to the anecdotal reports noted above (Kell, Lubinski, Benbow, \& Steiger, 2013).

Consistent and robust correlations have been reported between spatial visualization skills and a breadth of mathematical tasks (Mix \& Cheng, 2012). For example, spatial visualization skills have been linked to performance in geometry (Delgado \& Prieto, 2004), algebra (Tolar, Lederberg, \& Fletcher, 2009), numerical estimation (Tam, Wong, \& Chan, 2019), word problems (Hegarty \& Kozhevnikov, 1999), 


Task Description
3D Mental Rotation
"Which two figures are
identical to the target figure
on the left (just seen form
different angles)?"

Fig. 1 Examples of measures used to capture individual differences in spatial visualization skills

mental arithmetic (Kyttälä \& Lehto, 2008), and advanced mathematics (e.g., function theory, mathematical logic, computational mathematics; Wei, Yuan, Chen, \& Zhou, 2012). Figure 1 presents a few examples of the types of measures that are typically used to capture individual differences in spatial visualization skills. In subsequent sections, we return to the question of what it is about this type of reasoning that might explain the consistent correlations with mathematics, generally, and with numerical reasoning more specifically.

As mentioned above, this review targets candidate mechanisms that link spatial visualization skills and basic numerical competencies. By basic numerical competencies we are referring to skills that relate to a basic understanding of number symbols and their various relations and applications (see Fig. 2 for examples). For example, tasks that require participants to compare and order numbers, perform arithmetic, and answer numerical word problems make up the sort basic numerical competencies we are referring to. Hereafter, the term mathematical and numerical reasoning will be used to refer this collection of tasks. The decision to specifically target numerical reasoning skills and not mathematics more generally was done for two reasons: First, in an effort constrain the literature search, and second, because the relationship between spatial visualization and numerical skills is not overtly obvious. While many branches of mathematics are inherently spatial, including geometry and measurement, the same cannot so easily be said of the most basic of mathematical entities and operations: numbers and arithmetic. Indeed, the question of why spatial visualization skills are linked to basic numerical competencies remains poorly understood. This review aims to provide insight into this question. We begin our review of the four accounts of why spatial visualization and numerical reasoning might be linked by considering the possibility that numbers may be characteristically spatial.

\section{Spatial representation of numbers account}

Numbers are the building blocks of mathematics: Their use omnipresent and fundamentally linked to almost all branches of mathematics. For this reason, any association between spatial processing and numbers is of potential critical importance in the effort to better understand the robust link between spatial skills and mathematics performance. As reviewed next, 


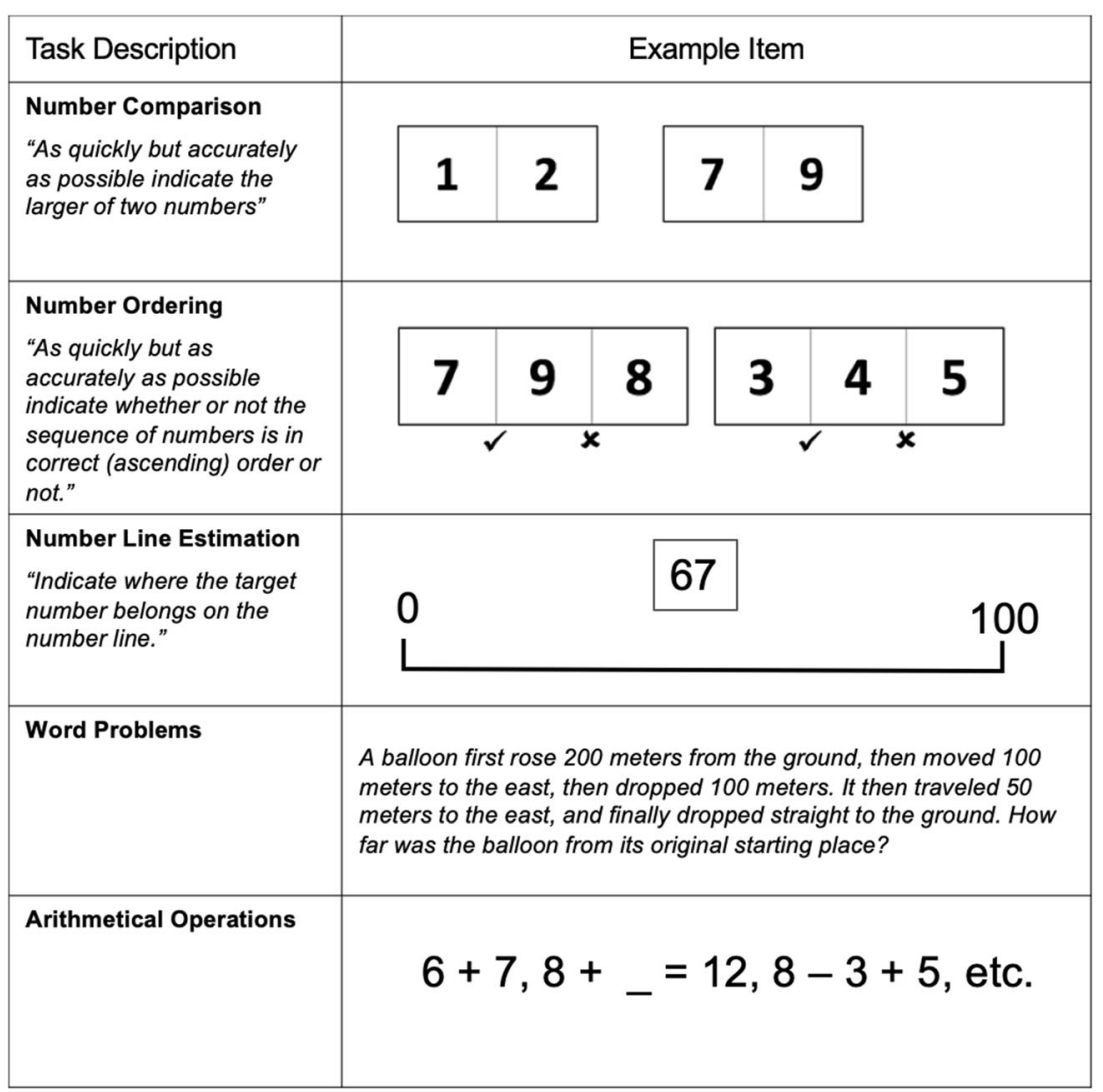

Fig. 2 Examples of measures used to capture individual differences in numerical reasoning

there is a substantial body of research indicating that numbers may be represented spatially. According to a recent study on the subject, "spatial processing of numbers has emerged as one of the basic properties of humans' mathematical thinking" (Patro, Fischer, Nuerk, \& Cress, 2016, pp. 126).

However, it remains unclear whether and to what extent spatial representations of number may confer any advantages to learning and doing mathematics. Moreover, and most germane to the purposes of the current review, it is not well understood what role higher-level spatial skills, namely spatial visualization skills, may play in the spatial representation of numbers.

The idea that numbers might be represented spatially has origins in Sir Francis Galton's mental imagery studies of the late 1800s (Galton, 1881). Galton provided the first evidence to suggest that numbers may be conceived as objects corresponding to specific positions in space:

Those who are able to visualize a numeral with a distinctness comparable to reality, and to behold it as if it were before their eyes, and not in some sort of dreamland, will define the direction in which it seems to lie, and the distance at which it appears to be. If they were looking at a ship on the horizon at the moment that the figure 6 happened to present itself to their minds, they could say whether the image lay to the left or right of the ship, and whether it was above or below the line of the horizon; they could always point to a definite spot in space, and say with more or less precision that that was the direction in which the image of the figure they were thinking of first appeared. (1881, pp. 86)

Galton referred to such visualizations as number forms, noting that people's descriptions of such visualizations varied according to their visual-spatial properties, including differences in orientation, colour, and brightness. (e.g., see Fig. 3). Despite such differences, number forms were said to represent a reliable and stable trait within individuals.

Galton's studies on number forms is important because it provided the first evidence that people may represent numbers in a spatial format; most typically from left to right, akin to an actual number line. During the last several decades, considerable research efforts have followed up on this possibility through a wide assortment of empirical investigation. Perhaps the most influential study in this regard is Dehaene et al.'s (1993) original findings on the SNARC effect (SpatialNumerical Association of Response Codes). In brief, the SNARC effect refers to the finding that people tend to automatically associate smaller numbers (e.g., 1, 2,3) to the left side of space and larger numbers (e.g., 7, 8, 9) to the right side of space. People are faster and make fewer errors when 


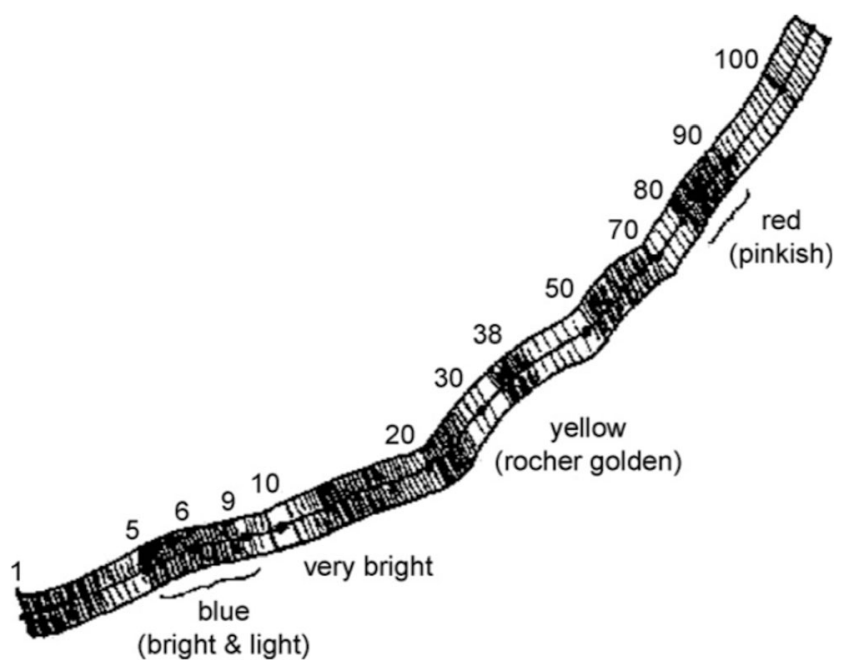

Fig. 3 An example of how one of the participants in Galton's study described their visualization of numbers

making parity judgments (i.e., determine whether a number is even or odd) when using the left hand to make judgements about small numbers and use the right hand to make judgements about larger numbers. This finding has been interpreted as evidence for the existence of a mental number line: A metaphor used to describe the tendency for individuals form Western cultures to conceive numbers as ordered magnitudes along a left-to-right axis. Indeed, the mental number line has been theorized to underlie a host of studies examining spatialnumerical associations (SNAs; e.g., see Toomarian \& Hubbard, 2018). For example, line bisection tasks (Calabria \& Rossetti, 2005), spatial attention tasks (Fischer \& Fias, 2005), and even random number generation are but a few examples of tasks said to reveal spatial-numerical biases, interpreted as support for the presence of a "mental number line" (Loetscher, Bockisch, Nicholls, \& Brugger, 2010). Arithmetic processing has also been suggested to induce automatic spatial-numerical biases (Knops, Viarouge, \& Dehaene, 2009). For example, the operation-momentum effect refers to findings of left-right biases associated with addition and subtraction. Adult participants tend to overestimate answers to addition problems and underestimate answers to subtraction problems (McCrink, Dehaene, \& DehaeneLambertz, 2007). Even when no calculation is required the mere presence of the operators themselves (i.e., + and -) have been found to influence left-right spatial biases (Mathieu et al., 2017). Importantly, evidence suggests that SNAs are mediated through cultural and educational practices. For example, the SNARC effect is reversed in cultures that read from right to left (Shaki, Fischer, \& Petrusic, 2009). Taken together, there is considerable evidence to suggest that numerical thinking is related to spatial biases. These biases, in turn, have been taken as evidence of the "mental number line."

Critically, the mental number line has been posited to underlie both automatic/unconscious processing of numbers as well as more effortful/conscious processing of numbers (Fischer \& Fias, 2005; Schneider et al., 2018; Toomarian \& Hubbard, 2018). As we now demonstrate, this distinction has important implications in addressing the question of when and why spatial skills and numerical reasoning are related. While Galton's inquiries centered around conscious visualizations of number, the vast majority of studies on SNAs have examined automatic numerical-spatial biases. Research on the latter has revealed little evidence that SNAs are related to individual differences in numerical reasoning skills (Cipora, Patro, \& Nuerk, 2015). Although a systematic review is needed to more fully investigate these relations, it is reasonable to conclude that automatic spatial-biases (as measured with the SNARC effect for example) have little influence on higher level numerical and mathematical processing. There is even some evidence to suggest that a negative association may exist. Practising mathematicians, for example, have been found to demonstrate weaker numerical-spatial biases compared to control subjects (Cipora et al., 2016). These findings stand in stark contrast to the research literature on intentional spatial-numerical associations (e.g., see Schneider et al., 2018).

For example, research on the number line estimation task reveals a consistent and reliable association between performance on the task and numerical reasoning. (Schneider et al., 2018). People who are more accurate at estimating where a given number belongs on a horizonal line flanked by two end points (e.g., 0 - 100; see Fig. 2), tend to also demonstrate better numerical and mathematical reasoning skills. Results of recent meta-analysis revealed an average correlation of .44 between number line task performance and mathematics (counting, arithmetic, school mathematics achievement) across the ages of $4-14$ years $(\mathrm{N}=10,576$; Schneider et al., 2018). This effect size is considerably larger than the correlations that have been reported between other foundational numerical skills and mathematics achievement. For example, measures of symbolic number comparison - a widely accepted measure of numerical fluency - is estimated to share a .30 correlation with mathematics achievement (e.g., see Schneider et al., 2017). Moreover, to date, the most effective mathematics interventions have used the number line as the instructional tool used to enhance students' numerical reasoning (Fischer, Moeller, Bientzle, Cress, \& Nuerk, 2011; Link, Moeller, Huber, Fischer, \& Nuerk, 2013; Ramani \& Siegler, 2008). Interestingly, number-line training studies are theorized to be effective because they lead to a more refined "mental number line" (Fischer et al., 2011; Siegler \& Ramani, 2009).

Thus, in considering the above finings, we are left with an interesting paradox. Automatic/unconscious spatial-numerical associations do not appear to be related to individual differences in mathematics. On the contrary, intentional spatialnumerical associations appear to be strongly related to individual differences in mathematics. Moreover, both types of 
spatial-numerical associations - the unconscious and the conscious - are said to reflect the "mental number line." What might explain this disconnect?

To gain insight into this question, we turn to the role that spatial visualization may play in first forming spatialnumerical associations. Several studies have now provided evidence that spatial visualization skills relate to improved number line performance, which in turn is related to improved arithmetic and mathematics performance (Gunderson, Ramirez, Beilock, \& Levine, 2012; LeFevre, Jimenez Lira, Sowinski, Cankaya, Kamawar, \& Skwarchuk, 2013; Tam, Wong, \& Chan, 2019). In other words, linear numerical representations have been found to mediate relations between spatial and numerical reasoning. Other researchers have found that spatial visualization skills are positively correlated to automatic SNAs, including the SNARC effect (Viarouge, Hubbard, \& McCandliss, 2014). It has been hypothesized that strong spatial visualization skills underlie a greater ease and fluency in which one can move up and down and carry out arithmetical operations along the mental number line (Viarouge, Hubbard, \& McCandliss, 2014). However, this finding is somewhat at odds with the evidence viewed above. That is, if spatial visualization skills are linked to automatic SNAs, might we also expect automatic SNAs to relate to mathematics? Currently, it remains unclear whether, how, and why automatic SNAs mediate relations between spatial visualization and mathematics.

While it is easy to imagine the role that spatial visualization skills play in tasks that explicitly call upon the need to map numbers to space (e.g., the empty number line task), it is more difficult to imagine why spatial visualization skills are associated with automatic SNAs. One possibility is that automatic SNAs are an artefact of numerical-spatial relations formed earlier in development. That is, early in development, spatial visualization skills may help children to construct relations between space and number. Over time, children may internalize these spatial-numerical relations, a process that eventually gives rise to automatic numerical-spatial biases. An important question is whether spatial visualization skills are still related to automatic SNAs, once the "building process" is complete. While the study by Viarouge et al. (2014) suggests that the answer to this question is yes, this is the one and only study to directly address this question, to our knowledge. Moreover, even if follow-up research confirms relations between spatial visualization skills and automatic SNAs, we are still left with the question of why conscious SNAs but not automatic SNAs relate to mathematics.

One plausible explanation, related to the argument above, is that the intentional mapping of numbers to space involves a host of mathematical reasoning skills, including spatial and proportional reasoning (Barth \& Paladino, 2011; Gunderson et al., 2012; Simms, Clayton, Cragg, Gilmore, \& Johnson, 2016). Although more automatic mappings of number to space may also require these same skills, their influence on task performance may not be as paramount. Accordingly, the relation between spatial-numerical mappings and mathematics may be explained in part due to the extent that other mathematical relevant processes, including spatial visualization, are recruited during task execution. Said differently, mapping tasks that require higher levels of mathematical reasoning and precision are expected to share higher correlations with mathematical tasks that also require these same higher levels of precision and mathematical reasoning. This is a somewhat straightforward prediction, and, notably, one that aligns well with the spatial modeling account, but has yet to be investigated. As discussed in the next section, it is also possible that automatic SNAs are not as automatic as they appear, but rather constructed on the fly, within the confines of working memory and dependent on the specific task demands.

Critically, the mapping of numbers to space - by way of a mental number line - might be but one example in which spatial visualization skills are used to map and make sense of numerical-spatial relations (e.g., see Lakoff \& Núñez, 2000; Marghetis, Núñez, \& Bergen, 2014). As pointed out earlier, mathematics is full of examples in which numbers are mapped to space (e.g., geometric proofs, measurement, topology, etc.). Might spatial visualization skills play a more general role in mapping numbers, but also other mathematical entities and concepts, onto space? Indeed, as discussed earlier, the relationship between spatial visualization skills extends to a wide variety of mathematical tasks (Mix \& Cheng, 2012). Moreover, numbers do not appear to be unique in their automatic association of left-right biases. For example, the SNARC effect has been extended and replicated with other ordered stimuli such as the days of week, months of the year, and letters of the alphabet (Gevers, Reynvoet, \& Fias, 2003, 2004). Relatedly, the SNARC effect appears to be flexible and prone to priming effects. For example, Fischer et al. (2010) trained participants to view large numbers on the left and small numbers on the right and found evidence of a reversed SNARC effect (Fischer, Mills, \& Shaki, 2010). Together, these findings suggest that the SNARC effect is (1) not limited to numbers, and (2) easily modulated by context. These findings have led to the hypothesis that the SNARC effect is indicative of context-dependent mappings between ordered stimuli (numbers, months, letters) and space.

Moreover, these findings challenge the long-held belief that numbers are inherently spatial and automatically associated with space. Instead, an alternative viewpoint has emerged, positing that numerical-spatial associations are constructed in working memory during task execution (van Dijck $\&$ Fias, 2011). Whether or not spatial visualization plays a role in this online constructive process remains to be studied. However, given the close link between spatial visualization skills and explicit numerical-spatial mappings (i.e., number line estimation tasks), spatial visualization skills may also facilitate more covert numerical-spatial mappings. 
Taken together, questions remain regarding the extent to which numbers are automatically associated with space versus actively constructed on a moment-to-moment basis. Moreover, the role of spatial visualization in mapping numbers to space remains largely unknown. In the next section, we continue to expand on the central idea presented in this section - that is, spatial and numerical skills may be linked because numbers are represented spatially. While this section has revealed behavioral evidence in favor of a close coupling of numbers and space, the next section addresses questions about the neural mechanisms that underlie these relations.

\section{Shared neural processing account}

According to the shared neural networks account, spatial and numerical processing may be related because they rely on the same brain regions and make use of similar neural computations. The first indication that this may be the case came from neurological case studies. Individuals with damage to the parietal lobes were sometimes observed to demonstrate joint deficits in both spatial and numerical processing (Gerstmann, 1940; Holmes, 1918; Stengel, 1944). In fact, Gerstmann's Syndrome presents a rare but specific example of how damage to the parietal lobes (i.e., the left angular gyrus) is associated with impaired spatial and numerical reasoning. People with Gerstmann's Syndrome typically display a tetrad of symptoms including acalculia, left-right confusion, finger agnosia (difficulty identifying one's fingers), and dysgraphia (difficulty with writing) (Gerstmann, 1940). It has been suggested that these difficulties may be due to a more general deficit in the mental manipulation of visual-spatial images, including impaired mental rotation skills (e.g., see Mayer et al., 1999).

Research on patients with hemi-spatial neglect provides further evidence that space and number may depend on intact parietal lobes. Individuals with hemi-spatial neglect demonstrate an inability to attend to the contralesional portion of space (e.g., inability to attend to the left side of space when the lesion is in the right parietal lobe). This condition is associated with a skewed ability to indicate the mid-point of both real and imagined objects, but also the mid-point of numerical intervals (Bisiach \& Luzatti, 1978; Zorzi et al., 2002). For example, Zorzi et al. (2002) asked right-brain-damaged patients to indicate the mid-point of two spoken numbers, such as "two" and "six." Presumably, due to an impaired mental number line, patients tended to overestimate the midpoint between two numbers as the interval between them increased (e.g., stating "five" as the midpoint between "two" and "six."). Taken together, neuropsychological case studies provide the earliest evidence that spatial and numerical processing may rely on common parietal cortex.
More recently, the advent of fMRI has given way to a host of follow-up investigations into the neural correlates of numerical and spatial thinking. This body of research points to the intraparietal sulcus (IPS) as the critical juncture in which numbers and space may interact (e.g., see Hawes, Sokolowski, Onoyne, \& Ansari, 2019; Fig. 4). Indeed, it is now well established that the IPS and its neighboring regions play a critical role in reasoning about a variety of magnitudes, including non-symbolic quantities, space (size and shape), luminance, and even abstract notions such as number and time (see Kadosh, Lammertyn, \& Izard, 2008; Hawes et al., 2019b; Walsh, 2003). Thus, there is evidence to suggest that basic spatial and numerical processes rely on common regions in and around the IPS.

There is also evidence that higher-level spatial skills, such as mental rotation, may also draw on these same parietal regions. For example, it has long been recognized that a central function of the parietal lobes is the performance of spatial transformations. Support for this can be seen in the results of a meta-analysis by Zacks (2008) on the neural correlates of mental rotation. Zacks found evidence found evidence to suggest that the IPS was the most robust and consistently activated brain region associated with mental rotation. Other spatial visualization processes, such as being able to compose/decompose and translate geometric shapes, have also been associated with activity in this region (Jordan, Heinze, Lutz, Kanowski, \& Jäncke, 2001; SeydellGreenwald, Ferrara, Chambers, Newport, \& Landau, 2017). One reason that spatial and numerical reasoning may be linked is through shared processes related to mental transformations. According to Hubbard et al. (2009): "parietal mechanisms that are thought to support spatial transformation might be ideally suited to support arithmetic transformations as well' (2009, pp. 238). Indeed, this is an intriguing possibility and one that supports the neuronal re-cycling hypothesis.

According to the neuronal recycling hypothesis, numbers as well as other mathematical symbols and concepts may reuse the brain's neural resources that were originally specialized for interacting with the physical world (e.g., see Anderson, 2010, 2015; Dehaene \& Cohen, 2007; Lakoff \& Núñez, 2000; Marghetis, Núñez, \& Bergen, 2014). In other words, numerical processing may co-opt or re-use the brain's more ancient and evolutionary adaptive spatial and sensorimotor systems, which originally served our abilities to interact with tools, objects, and locations in space (Dehaene et al., 2003; Johnson-Frey, 2004; Lakoff \& Núñez, 2000). Marghetis et al. (2014) offer this summary of the neuronal re-cycling account: "we may recycle the brain's spatial prowess to navigate the abstract mathematical world" (pp. 1580). The neuronal recycling hypothesis has been used by many as an explanation for numerical-spatial biases observed through both behavioral and neuroimaging studies.

Taken together, there is compelling evidence that spatial and numerical processing are associated with overlapping 
Symbolic Number

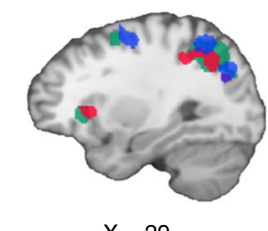

$X=29$

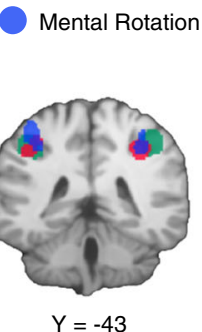

$Y=-43$

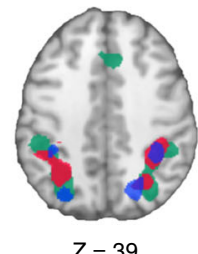

$Z=39$
Mental Arithmetic

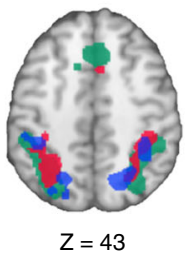

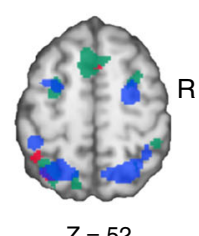

$Z=52$

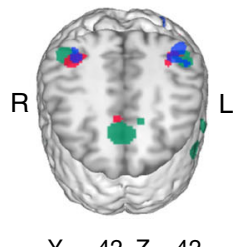

$Y=-42 \quad Z=42$

Fig. 4 Meta-analysis of fMRI studies examining brain regions associated with mental arithmetic (green), basic symbolic processes (red), and mental rotation (blue)

regions of the parietal cortex, namely in and around the IPS. However, there are also some notable gaps in the literature. One such gap is the emphasis placed on uncovering how basic spatial processes (e.g., comparing line lengths) relate to basic numerical processes (e.g., comparing Arabic digits; e.g., see Sokolowski, Fias, Mousa, \& Ansari, 2017). To date, research on higher-level spatial skills (i.e., those that require spatial transformation, such as mental rotation) have been studied in isolation from neuroimaging studies of numerical cognition (but see Hawes et al., 2019b). So, although there is good evidence to suggest that higher-level spatial skills also rely on processes associated with the IPS, we do not yet have any direct evidence (i.e., from a single study) for this correlation. However, this is a critical gap in the literature for reasons discussed earlier. While there is robust evidence for relations between spatial visualization skills and numerical and mathematical performance, there is little evidence that spatial representations of number relate to individual differences in numerical and mathematical performance. Thus, when it comes to better understanding individual differences in mathematics performance, much can be gained by studying the neural relations between spatial skills proper and numerical and mathematical reasoning.

\section{Spatial modeling account}

According to the spatial modeling account, spatial visualization is related to numerical reasoning because it provides a "mental blackboard" of which numerical relations and operations can be modeled and visualized. More specifically, spatial visualization has been posited to play a critical role in how one organizes, models, and ultimately conceptualizes novel mathematical problems (Ackerman, 1988; Mix et al., 2016; Uttal \& Cohen, 2012). Although there may be little to no need to model familiar mathematical content, such as memorized arithmetic facts, the visualization process may prove beneficial when confronted with novel mathematical content, such as arithmetic questions that require multi-step calculations. Moreover, the spatial modeling account functions to bridge past, present, and future knowledge states. For example, to solve the question $58+63$, one might use prior knowledge of arithmetic facts to arrive at a previously unknown arithmetic fact (e.g., reason that $50+60=110$ and $8+3=11$; therefore, the solution is $110+11=121$ ). To do this - bridge prior knowledge with newly created knowledge - one must also maintain the problem and interim solutions in mind. Whether or not these same functions might just as easily be ascribed to a working memory account is an important question and one we further address below.

Arguably, the most impressive feature of the spatial modeling account, but also perhaps its Achilles heel when it comes to empirical study, is that there are few, if any, limitations on the type of mathematical content that can be modeled by way of spatial visualization. Indeed, spatial visualization processes provide a space in which one can move back and forth between a multitude of representations; between the concrete and the abstract, the symbolic and the nonsymbolic, the real and the imagined, and static and dynamic representations (Antonietti, 1999). In short, there appear to be few limitations on the types of mathematical relations that can be modeled through visualizations. It is for this reason that it can be difficult to empirically investigate the spatial modeling account. How does one reveal the specific type of spatial modeling that occurs in the "mind's eye" of any given individual? Are some types of spatial modeling more conducive to effective mathematical reasoning than others?

One promising approach to these questions comes from studying how children model solutions to mathematical word problems. For example, Hegarty and Kozhevnikov (1999) presented children with the following word problem:

\section{"A balloon first rose 200 meters from the ground, then moved 100 meters to the east, then dropped 100 meters. It then traveled 50 meters to the east, and finally dropped straight to the ground. How far was the balloon from its original starting place?"}

Children's accompanying drawings to the problem revealed key insights and differences into how children modeled/visualized the problem. While some children's drawings were literal representations of the problem, others were 
more abstract and contained only the relevant mathematical details needed to answer the question. Based on these differences, children's drawings were categorized as either pictorial (more literal in representation) or visual-schematic (more abstract in representation; emphasis on relevant numericalspatial relations; see Fig. 5 for an example). Children who produced visual-schematic representations were more likely to arrive at the correct solution. Moreover, children who produced visual-schematic representations were also found to demonstrate significantly higher spatial visualization skills. Several studies have since replicated this finding (see Boonen, van der Schoot, van Wesel, de Vries, \& Jolles, 2013; Boonen, van Wesel, Jolles, \& van der Schoot, 2014). Taken together, these studies suggest that spatial visualization skills may indeed help learners to better model mathematical relations, which in turn, may lead to improved mathematical performance.

In the above studies on word problems, it appears best to create mental models of only the relevant mathematical details. However, the question of what to model is likely task/question specific. For some maths problems, it is not so much about "doing away" with irrelevant details, but about retaining, manipulating, and forming new relations with the information given. For example, take missing term problems, such as $5+\ldots=7$. It has been suggested that one of the ways in which children come to develop fluency with such questions is through the ability to re-structure (re-model) the problem. So, instead of $5+\ldots=7$, the learner might transform the question into the more familiar form, $\ldots=7-5$. What role might spatial visualization skills play in this process? To investigate this question, Cheng and Mix (2014) carried out a randomized controlled trial with 6- to 8-year-olds. Half the children were assigned to mental rotation training condition and the other half were assigned to an active control group. Compared to the control group, children in the mental rotation group demonstrated significant gains on the missing term problems. Consistent with the spatial modeling account, the authors suggested that gains on the missing term problems may have a resulted from an improved ability to re-model the problems into an easier format. This study provided the first causal evidence that spatial visualization training may transfer to mathematics. However, a recent follow-up study by Hawes et al. (2015) failed to replicate this finding. It is clear that more research is needed before causal claims can be made about the generalizability of spatial training to mathematics. In moving forward, such efforts should also try to more specifically address the mechanism of transfer. For example, what evidence is there that the changes in mathematics occur because of the effect that spatial training has on the way the problems are modelled? Insights into this question are needed in order to test the validity and make causal claims about the spatial modeling account.

As mentioned earlier, one of the predictions of the spatial modeling account is that spatial modeling is most used when dealing with novel versus familiar mathematical content. There is some evidence that this may be the case. To test this possibility, Mix et al. (2016) examined the relation between spatial skills, including spatial visualization, and novel and familiar mathematical content. Their results suggested that spatial skills were most closely related to novel mathematical problems. A follow-up study by Hawes et al. (2019a) provides additional insights into this issue. Using latent variable analyses, it was found that spatial visualization skills were highly correlated to both basic numerical skills as well as more advanced numerical skills (e.g., applied number problems, number operations). However, the relations between spatial visualization skills and higherlevel numerical skills were much stronger than relations between spatial visualization skills and basic numerical skills. These studies provide some important preliminary support for the spatial modeling account. However, these studies do not provide any direct evidence that spatial visualization is differentially used as a function of problem familiarity or difficulty.

It is important to note that the spatial modeling account overlaps with other theories of numerical and mathematical cognition. In particular, it bears close resemblance with grounded and embodied accounts of mathematical cognition a

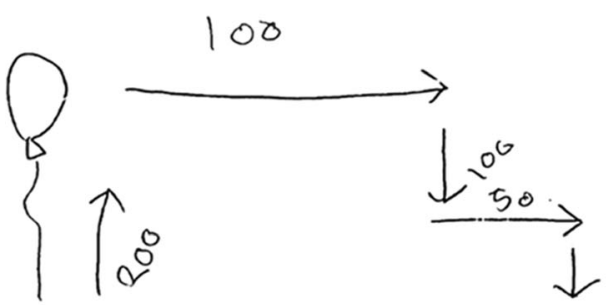

b

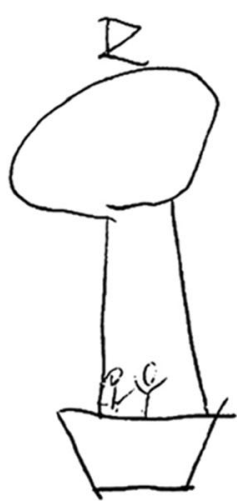

Fig. 5 An example of a visual-schematic representation (A) vs. a pictorial representation (B) 
(Lourenco et al., 2018). According to these perspectives, mathematical thought is grounded in our everyday sensory and bodily experiences (Anderson, 2010, 2015; Lakoff \& Núñez, 2000; Marghetis, Núñez, \& Bergen, 2014). It is through engaging with metaphors, mental imagery, and simulated actions that mathematics becomes meaningful, and ironically, "groundless" (e.g., see Lakoff \& Núñez, 2000). This view contrasts with the perspective that mathematics is largely independent of sensorimotor experiences and instead is a function of symbolic amodal thought (e.g., see Barsalou, 2008). Most relevant to the spatial modeling account is the role that mental simulation has been hypothesized to play in cognition in general, and in mathematics, in particular (Anderson, 2016; Barsalou, 2008; Huttenlocher, Jordan, \& Levine, 1994). Indeed, mental simulation and mental modeling are alike in that they describe mental processes related to the re-enactment of sensorimotor experiences (e.g., mental imagery) in the service of a future goal (e.g., arriving at the correct solution to a word problem). The following provides an apt summary of the grounded cognition account, including clear parallels with mental simulation and the spatial modeling account:

Operations with some of the objects in mental models are like operations with physical objects. In reasoning about these objects, the person mentally moves about on them or in them, combines them, changes their sizes and shapes, and performs other operations like those that can be formed on objects in the physical world (Greeno, 1991, pp. 178).

To be clear, the spatial modeling account is a more specific instantiation of mental simulation; one that is confined to the discipline of mathematics and deals explicitly with spatial relations. The above quote speaks to the "neuronal recycling" hypothesis mentioned earlier, offering additional insights into why space and number might both heavily recruit bilateral regions in and around the IPS. It is possible that numbers and various other mathematical concepts are processed in ways similar to the planning and actions associated with our handling of everyday objects. This point perhaps speaks to the common practice amongst mathematicians to refer to numbers as well as other mathematical concepts and abstractions as objects (Font, Godino, \& Gallardo, 2013). A better understanding of why and how mathematicians come to view various concepts as objects may prove useful in better understanding the spatial modeling account as well as the development of mathematical expertise. For example, decades of research on human learning and memory indicate that objects are more easily remembered and expressed than abstract concepts (Paivio, 1983, 2013). Might this same finding apply to the realm of mathematics? Rather than dealing with isolated fragments of mathematical procedures carried out in a stepby-by fashion, presumably under the control of a more verbally mediated cognitive system, might the mathematical mind operate with greater ease and efficiency when dealing with holistic and object-like mental models? At the moment, research into these as well as other related questions concerning the spatial modeling account remain scarce. As such, the spatial modeling account remains a largely speculative account of why spatial visualization and numerical reasoning are so often linked.

\section{Working memory account}

Another way in which spatial visualization and numerical skills may be related is through another variable which shares relations with performance in both of these areas. For example, it is possible that spatial visualization skills are essentially a proxy for other cognitively demanding skills, such as executive function skills, working memory, and general intelligence. Visual-spatial working memory (VSWM), in particular, may explain the relations between spatial visualization and numerical skills. In this section, we review the evidence for and against this proposal.

Research to date suggests that both spatial visualization skills and VSWM are strongly related to numerical reasoning. For example, performance on spatial visualization tasks, such as mental rotation, have been linked to basic measures of numerical competencies, including arithmetic, number comparison, and number line estimation. Similarly, VSWM has also been found to explain similar amounts of variance in these same measures. Furthermore, there is evidence of close relations between all three of these variables - VSWM, spatial visualization, and numerical reasoning - when measured concurrently in the same studies (Alloway \& Passolunghi, 2011; DeStefano \& LeFevre, 2004; Hawes et al., 2019a; Kaufman, 2007; Kyllonen \& Christal, 1990; Kyttälä et al., 2003; Li \& Geary, 2017; Mix et al., 2016). Together, these findings question the extent to which spatial visualization and VSWM skills make unique contributions to numerical abilities.

It has been suggested that poor spatial abilities are a result of low VSWM. For example, several researchers have demonstrated notable differences in people of low- versus highspatial abilities in their abilities to form, maintain, and transform visual-spatial representations (Carpenter \& Just, 1986; Just \& Carpenter, 1985; Lohman, 1988). Carpenter and Just (1986) concluded that "a general characterization...is that low spatial subjects have difficulty maintaining a spatial representation while performing transformations" (p. 236). That is, individuals with low-spatial abilities tend to "lose" information as they engage in the act of spatial transformation. For example, when mentally rotating cube figures, individuals with low-spatial abilities often lose "sight" of the mental image and require multiple attempts at rotation (Carpenter \& Just, 1986; Lohman, 1988). Against this background, 
researchers have attributed individual differences in spatial visualization as primarily due to differences in working memory (e.g., see Hegarty \& Waller, 2005).

Evidence to suggest that spatial visualization skills and VSWM are not as related as suggested above comes from three separate bodies of research: factor analyses, research on sex differences, and training studies. Studies from factor analytic studies suggest that VSWM, spatial visualization, and executive functions represent distinct cognitive constructs (i.e., latent variables; Hawes et al., 2019a; Miyake et al., 2001). Moreover, Hawes et al., 2019a demonstrated spatial visualization and numerical skills (both basic and advanced) not only represent distinct constructs, but that the relations between the two could not be explained by general intelligence or executive functions, including measures of VSWM. Lastly, Mix et al. (2016) found evidence that in sixth grade, VSWM shared stronger cross-loadings with a general mathematics factor compared to measures of spatial visualization, which were more associated with a general spatial factor. Together, these findings indicate that spatial visualization and VSWM represent separable cognitive factors and share differential relations with numerical and mathematics performance.

Further evidence that spatial visualization and VSWM are separable constructs can be gleaned from findings of reliable sex differences on measures of spatial visualization but not VSWM (Halpern et al., 2007). ${ }^{1}$ Beginning by about the age of 10 years, males tend to outperform females on measures of mental rotation, with estimated effects sizes ranging from .91.0 (Halpern et al., 2007; Titze, Jansen, \& Heil, 2010). Importantly, sex differences are not confined to mental rotation tasks but also emerge on other spatial visualization tasks, including mental paper folding tasks (Halpern et al., 2007). Findings of sex differences in spatial visualization skills, but not VSWM, further suggests that these two aspects of visualspatial processing may represent distinct constructs.

Training studies provide further evidence that VSWM and spatial skills behave and operate in unique ways. Although the effects of VSWM training are hotly debated and there is little evidence that training generalizes to other untrained tasks (e.g., mathematics; Redick, Shipstead, Wiemers, MelbyLervåg, \& Hulme, 2015), a different picture has emerged with respect to spatial training. A recent meta-analysis of $217 \mathrm{spa}-$ tial training studies by Uttal et al. (2013) indicates that spatial thinking can be improved in people of all ages and through a wide assortment of training approaches (e.g., course work,

\footnotetext{
$\overline{1}$ It should be recognized that this argument also applies to relations between spatial visualization and numerical reasoning. Although sex differences are frequently observed on measures of spatial visualization (namely mental rotation), sex differences do not regularly occur on measures of numerical reasoning (e.g., see Hutchison, Lyons, \& Ansari, 2019; Kersey, Braham, Csumitta, Libertus, \& Cantlon, 2018). This finding provides an additional constraint to consider in the attempt to disentangle the link between spatial visualization and numerical reasoning.
}

task-based training, video games). Furthermore, the researchers concluded that although further evidence is still required, it appears as though the effects of spatial training transfer to a variety of novel and untrained spatial tasks. In subsequent sections, we return to the topic of spatial training and the extent to which spatial training transfers to numerical reasoning. The take away point in this section, however, is that compared to VSWM, spatial visualization skill appears to represent a more flexible and adaptive cognitive system, providing further insight into the separability of VSWM and spatial skills.

At this point, it is worth returning to the question at hand: Does VSWM explain the relationship between spatial visualization skills and numerical/mathematical abilities? Based on the available evidence, there are reasons to suspect that (1) spatial visualization and VSWM are separable constructs, and (2) that each share independent pathways with numerical skills. An important follow-up question is why VSWM and spatial visualization skills may differentially contribute to numerical and mathematical learning and performance.

One proposal is that VSWM and spatial visualization differ according to the cognitive demands placed on the need to "recall" versus "generate" visual-spatial information. For example, at a measurement level, most VSWM measures primarily require participants to recall, maintain, and (sometimes) manipulate visual-spatial information. Most spatial visualization measures, on the other hand, require participants to perceive, maintain, manipulate, and generate visualspatial solutions. Thus, the shared need to maintain and manipulate visual-spatial information may explain the previously reported correlations between VSWM and spatial visualization. However, the differences in task requirements might be one reason to predict differential relations with numerical performance. While VSWM skills may play a greater role in numerical tasks that emphasize the need to recall and maintain information (e.g., basic arithmetic), spatial visualization skills may play a greater role in numerical tasks that emphasize the need to generate novel solutions (e.g., word problems, applied problems). Notably, this prediction supports the spatial modeling account discussed earlier. Spatial visualization skills are predicted to be especially useful, even more so than VSWM, on problems that require the modeling and generation of problem solutions. Future research is needed to formally test this hypothesis.

\section{Discussion}

To this point, the relationship between spatial ability and mathematics has been well-studied but scarcely understood. The purpose of this review was to shed light on this issue by reviewing the literature in search of potential mechanisms that might explain the historically tight relations between spatial 
and numerical reasoning. More specifically, this review targeted the ways in which spatial visualization might be linked to numerical reasoning. Based on comprehensive review of research from psychology, neuroscience, and education, four potential mechanisms were identified: (1) Spatial representation of numbers account, (2) shared neural processing account, (3) spatial modeling account, and (4) working memory account. In the following section, a brief summary of each account is provided. We then engage in a more thorough discussion of the limitations as well as potential for improving spatial-numerical relations.

\section{A summary of the four accounts}

In brief, the spatial representation of numbers account suggests that numbers and their various relations are represented along a "mental number line." In turn, the precision of one's mental number line has been posited to play an important role in performing a host of numerical reasoning tasks, including comparing, ordering, and operating on numbers. A small body of research suggest that spatial visualization skills play a fundamental role in the learning and formation of numerical representations. The shared neuronal processing account suggests that numbers and space are linked through shared underlying neuroanatomical substrate. According to the neuronal recycling theory, numerical reasoning capacities re-use the same neuronal resources that were originally (evolutionarily speaking) deployed for spatial reasoning, including spatial visualization. The spatial modeling account places much more emphasis on spatial visualization as a more general mechanism used to model, organize, and simulate a wide variety of numerical concepts. This account is closely connected to other theories of mathematical reasoning, including grounded, embodied, and the aforementioned neuronal recycling accounts. A common feature of these theories is the use of the visual-spatial imagination to act upon mathematical objects, including numbers, in ways not unlike the ways we experience and use objects in the real world. Lastly, the working memory account calls into question unique relations between spatial visualization and numerical reasoning skills. Instead, the link may have its roots in individual differences in visualspatial working memory (VSWM). However, evidence to date suggest that these two constructs are not one and the same and make independent contributions to numerical and mathematical performance. Moving forward, it will be important to continue to study the ways in which spatial visualization represents a unique construct as well as the ways in which it interacts with other cognitive systems.

\section{An integrated description of the four accounts}

The extent to which these various accounts are descriptions of the same underlying mechanism but in different forms and at different levels of analysis is an important question. For example, it is possible that one of the ways in which numbers become represented spatially is through the active processes of spatial modeling (e.g., visualizing a number line to reason about numerical relations). From a biological perspective, it could be that the IPS and closely associated regions provide the necessary neuronal networks to carry out these modeling and transformational processes. Moreover, even when the spatial modeling of numerical concepts no longer serves the individual (i.e., the concepts at hand have become automatized more or less), these same neural substrates may continue to underlie both numerical and spatial processes (e.g., see Hawes et al., 2019b). This may occur despite an independence in function. If we assume that spatial visualization is a relatively stable trait, then we should expect to see lasting correlations between spatial visualization and numerical skills even when spatial visualization no longer serves a purpose in one's semantic understanding/representation of number. In other words, spatial and numerical processes may continue to be correlated, both neurally and behaviorally, long after they have become conceptually divorced from one another. This relation may remain because of individual differences in spatial visualization skills that once helped give rise to conceptual mappings between numbers and space. This integrated account may explain why we continue to see correlations between spatial visualization skills and basic numerical competencies into adulthood. It might also explain why we see relations between intentional numerical-spatial mappings (e.g., as measured with the number line task) and mathematics (Schneider et al., 2018), but mixed evidence for relations between automatic numerical-spatial mappings (i.e., SNARC) and mathematics (Cipora, Patro, \& Nuerk, 2015).

From this example it can be seen how biology and behavior interact in complex ways to give rise to potentially dynamic and ever-changing numerical-spatial relations. To what extent does genetically endowed neuroanatomical structures influence one's abilities to visualize numerical-spatial relations? To what extent is spatial visualization malleable and transferable to numerical reasoning? These are important questions; the answers of which may help to more fully understand the interplay that may exist between the various accounts of space-number relations.

\section{Biological considerations of the four accounts}

An interesting question concerns the extent to which one's spatial visualization abilities are constrained by genetic and corresponding neuroanatomy. Results from a meta-analysis of twin studies ( $N=18,296$ monozygotic twins; $N=23,327$ dizygotic twins) suggest that spatial visualization abilities are largely heritable (.61), with non-shared environmental factors having a substantial impact (.43) and shared environmental factors have a little effect (.07; King, Katz, Thompson, \& 
Macnmara, 2019). In other words, approximately $60 \%$ of the variability in spatial visualization can be accounted for, statistically, by genetic differences between people (in this particular sample). Even more germane to the current study, however, is the extent to which relations between spatial visualization and numerical abilities are due to shared genetics. This question was recently addressed by Tosto et al. (2014) through a twin study ( $\mathrm{N}=1,539$ monozygotic twins; $\mathrm{N}=2,635$ dizygotic twins). As expected, they found a strong relation between spatial visualization skills and mathematical abilities, including measures of numerical reasoning $(r>.40)$. Moreover, they found that approximately $60 \%$ of this overlap was explained by common genetic effects, while $40 \%$ of the overlap was due to environmental experience ( $26 \%$ and $14 \%$ by shared and non-shared environments respectively). Taken together, these studies suggest genetics may help explain individual differences in spatial visualization skills as well as common variance between spatial and numerical relations.

\section{The malleability of spatial visualization}

Although biological factors may place certain constraints on one's range of spatial visualization abilities, it is also clear that spatial skills are highly malleable constructs (Uttal et al., 2013). Compared to other core cognitive capacities, including working memory, spatial abilities - most notably spatial visualization skills - appear to be highly subject to practice and training effects. Evidence for this comes from Utall et al. (2013) who performed a meta-analysis examining the overall effects of 217 spatial training studies over a 25 -year period (1984-2009). The study concluded that spatial training is an effective means for improving spatial thinking in people of all ages and across a variety of training techniques (e.g., video games, in-class training, spatial task training). The average effect size was large, approaching half a standard deviation (0.47). In theory, an improvement of this magnitude would approximately double the number of individuals with the spatial skills typically associated with being an engineer (Uttal et al., 2013). Moreover, the results revealed evidence of equal transfer for near and intermediate transfer measures. That is, training on one particular task, such as mental rotation, was found to not only lead to improvements in that same type of task, but resulted in improvements in untrained spatial tasks, such a mental paper folding (e.g., see Wright et al., 2008; Chu \& Kita, 2011). In terms of durability, similar gains were observed immediately after training, less than 1 week delay, or less than one month delay. Moving forward, it will be important to assess just how long training related gains persists. The results of this study are important but puzzling.

They are important as the implications are significant and far reaching, especially in considering the ways in which spatial training might help boost STEM-related performance (as suggested in the engineering example above). The results are puzzling in that the effects of training spatial abilities appears unlike the training of any other cognitive abilities. To our knowledge, only spatial training has been found to reliably yield intermediate transfer effects. Inquiries why this is and what makes spatial thinking an especially malleable cognitive construct are needed. This information may be useful in designing educational curricula and interventions.

\section{Does spatial visualization training transfer to mathematics?}

Given the evidence that spatial thinking is highly malleable, might spatial training be an effective means to improve numerical thinking? Indeed, the answers to this question have the potential to provide key insights into the four candidate mechanisms reviewed. Unfortunately, there is no conclusive answer to this question. To date, the evidence is mixed and appears to very much depend on the training approach. Moreover, there are only two studies which meet the criteria for a randomized controlled trial, both of which included small sample sizes. In Cheng and Mix's (2014) study, 58 children aged 6-8 years underwent either $40 \mathrm{~min}$ of spatial visualization training (mental rotation) or $40 \mathrm{~min}$ of an active control task (crossword puzzles). Compared to the control group, children in the spatial training group demonstrated gains on a measure of spatial visualization, but, most impressively, also demonstrated improvements on calculation problems of two types: standard calculation problems (e.g., $56+6=\ldots$ ) and missing term problems (e.g., $5+\ldots=12$ ). Gains were more pronounced on the missing terms problems. In line with the spatial modeling account, the authors suggested that the intervention may have been effective because it encouraged participants to more effectively model the problems (e.g., reorganize $5+\ldots=12$ into the more familiar question format, $=12-5 \cdot \overline{\text { A }}$ follow-up study by Hawes et al., 2015 failed to replicate these effects. In this study, 61 6- to 8-year-olds were assigned to either 6 weeks ( $3 \mathrm{~h}$ total) of computerized spatial visualization training program or 6 weeks of computerized literacy training (control). Compared to the control group, children who received spatial training demonstrated improvements on spatial visualization measures but demonstrated no evidence of gains on any of the mathematics measures, including miss-terming problems. These mixed findings and the small sample sizes used make it clear that much more research is needed before any conclusions can be made about whether spatial training generalizes to numerical and mathematical reasoning. Moreover, future studies of this sort should aim to more explicitly address the potential mechanism(s) that may or may not facilitate transfer. For example, to further test the possibility that the spatial modeling account might be at play (as the authors of both the training studies above suggest), it is imperative to capture evidence of this. This may be 
achieved through self-report strategy use or through having participants write or draw their solution strategies.

The two studies above represent the only randomized controlled studies; however, three other studies have examined the effects of spatial visualization training on mathematical performance through classroom-based "quasi-experimental" studies. Because random assignment did not occur at the level of the individual, the effects of these studies may have been more influenced by uncontrolled variables (e.g., different teachers). A major benefit of these studies, however, is that they were carried out by classroom teachers and may be considered more ecologically valid approaches to spatial training. In the first of these studies, Hawes, Moss, Caswell, Naqvi, \& MacKinnon, 2017 worked with kindergarten to Grade 2 teachers to implement a 32-week spatial visualization training intervention as part of teachers' regular mathematics instruction (total $\sim 45 \mathrm{~h}$ of spatial training). Compared to an active control group $(n=28)$, children in the spatial training classrooms $(n=39)$ demonstrated widespread improvement on a variety of spatial reasoning measures as well as gains on a symbolic number comparison task. However, as noted by the authors, many of the spatial visualization activities also incorporated aspects of numerical reasoning, which may have influenced the results. In fact, it is possible that the greatest potential for mathematical learning lies in the combination and integration of spatial and numerical instruction. However, such an approach limits the conclusions we can make about the unique contributions of spatial visualization in the learning of mathematics. In a somewhat similar study by Lowrie et al. (2017), the authors also found some evidence of transfer to mathematics following an intensive in-class spatial training program with 10 - to 12 -year-olds $(N=186 ; 20 \mathrm{~h}$ of training over 10 weeks). Children in the spatial training classrooms, but not the control classrooms, demonstrated improvements in spatial visualization as well as a comprehensive measure of mathematics. However, the mathematics measure included a combination of items related to both numerical concepts as well as geometrical concepts. Thus, it is possible that the gains were due to changes in geometrical reasoning, arguably closely related to or even dependent on spatial reasoning, and not numerical reasoning. Lastly, a recent study by Cornu et al. (2017) failed to find any transfer to mathematical reasoning. Compared to children in the control kindergarten classrooms $(n=57)$, children in the spatial training kindergarten classrooms $(n=68)$ demonstrated some gains on near transfer spatial measures, but showed no evidence of improvements on seven separate measures of mathematics (e.g., counting, number comparison, number naming, arithmetic).

In another quasi-experimental study, Cheung, Sung, and Lourenco (2019) examined the effects of an at-home spatial visualization intervention with 6 - to 7 -year-olds $(N=62)$. Compared to an active control group, children who participated in the at-home mental rotation training demonstrated near transfer gains in mental rotation ability and far transfer to arithmetic performance. Critically, such transfer could not be attributed to general cognitive improvement, as no gains were observed on measures of nonsymbolic comparison, verbal working memory, or language ability following training. Relevant to the current review, the authors speculate that far transfer may have been due to improvements in children's ability to mentally model arithmetical relations and/or ground numerical information along a mental number line.

Overall, the results of these "quasi-experimental" studies are difficult to interpret and at this point in time, few conclusions can be drawn. It is clear, however, that when improvements do occur in mathematics (and this was true in the Cheng and Mix study as well), the mechanism of transfer is not well understood. In fact, not one training study to date has systematically addressed the question of what might mediate the effects of spatial visualization training on numerical reasoning. Thus, moving forward, it will be critical to target the underlying agents of change. The four candidate mechanisms reviewed here provide a good place to start. For example, different predictions can be made depending on the different accounts reviewed. According to the spatial representation of numbers account, one might predict that spatial training is related to improvements in one's internal representation of numbers according to a more spatially precise mental number line. This refinement in one's "mental number line," in turn, is predicted to facilitate greater numerical reasoning. Critically, in order to test this hypothesis, future training studies will need to include measures of spatial-numerical mappings (e.g., intentional number-line estimation tasks, automatic SNA tasks, including SNARC effects). Any gains in more general measures of numerical reasoning should theoretically be mediated by change on these measures. As mentioned above, one way of testing the spatial modeling account would be to gain insights into the strategies that participants use while engaging in the numerical tasks. What evidence is there that the spatial visualization training actually led to an improved ability to mentally model the problem at hand? For example, collecting process data of the sort used in Hegarty and Kozhevnikov's (1999) word problem studies could be used to demonstrate the extent to which spatial visualization training results in improved schematic representations of the problems. Evidence of this sort would lend support for the spatial modeling account. In terms of the shared neural processing account, researchers have yet to examine the neural correlates of spatial training. However, a rather straightforward prediction would be that training-induced changes in neural activity (or the underlying neuroanatomical structures) should be correlated with improvements in numerical reasoning. Lastly, according to the working memory account, changes in spatial visualization should more broadly be encapsulated by changes in working memory. Indeed, it is possible that spatial visualization training is akin to working memory training. Future training 
studies thus need to also include measures of working memory to provide evidence for or against this possibility.

To conclude, future spatial training studies should look to go beyond simply measuring the effects of spatial training on numerical reasoning. Instead, trainings studies should be designed in ways that provide insights into the theorized mechanisms at play. This approach is critical in revealing why and under what conditions spatial training might be effective for some individuals but not others.

\section{Potential mediators and moderators}

As hinted at, the link between spatial visualization and numerical reasoning is likely to vary from individual to individual. For ease of clarification, this paper has only hinted at some of the potential mediators and/or moderators of the spacenumber relations. For example, we have suggested that spatial visualization may share stronger relations with unfamiliar vs. familiar numerical question types. With practice and experience, the need to engage visualization processes may be reduced. According to this proposal, the space-math link may differ across individuals as a function of their experience and familiarity with the mathematical task in question. For example, a child who is first learning basic arithmetic may find it useful to model the solution, whereas a child fluent in basic arithmetic may have no need to pause, reflect, and model the problem and solution. This suggests the need to not only consider the mathematical content under investigation, but also the learner's familiarity with the mathematical content when examining mechanisms underlying the space-math link. Said differently, mathematical experience may moderate relations between space and maths. To our knowledge, this represents a major gap in the literature and represents a promising area of future study.

In discussing the working memory account it was suggested that working memory or executive functions might mediate the relations between spatial visualization and mathematics. While research to date suggest evidence against this notion, more research is needed to more fully test this possibility. Moreover, it is possible that general intelligence ( $\mathrm{g}$ ) might account for the relations between space and mathematics. In fact, according to one account, intelligence might best be operationalized as the ability to spatially manipulate mental models (Lohman, 1996). Given prior findings of close relations between spatial visualization and general IQ (more specifically, nonverbal IQ), future research efforts are needed to further disentangle associations between spatial visualization abilities, general intelligence, and mathematics. To date, only one study has investigated this triad of relations and the results demonstrated strong and unique relations between spatial visualization and general mathematical abilities, even after controlling for g; Hawes et al., 2019a (as well as visual-spatial working memory). An important question moving forward is whether spatial visualization is related to mathematical reasoning due specific shared processes (e.g., the need to engage in mental rotation) or is related through more general processes (e.g., deductive reasoning). In addition to cognitive factors, many other variables might moderate the relation between spatial visualization and numerical reasoning, including, age, sex, type of mathematics instruction received in school (spatial vs. non-spatial), types of numerical reasoning required, past experiences with spatial learning, use of spatial versus non-spatial strategies, and various socioemotional and affective factors including spatial/mathematics anxiety. In combination, these factors represent a kaleidoscope of possible interactions. At present, we have only scraped the surface in studying how these and other variables may moderate space-number relations.

\section{Conclusion}

This paper highlights the potential ways in which spatial visualization and numerical abilities may be related to one another. Research is now needed to further probe and test the validity of these various accounts - both in isolation from one another but also in combination. Ultimately, it seems likely that all four accounts may offer insights into the ways in which spatial visualization and number are linked. In moving forward, it will not be enough to loosely base a study on one of the mechanistic accounts provided. For example, several studies to date have hypothesized strong relations between spatial abilities and mathematics because of research demonstrating shared neural resources. Indeed, as reviewed in this paper, there is good evidence to suggest that this is the case. However, we must go well beyond this level of theorizing: Not only stating which mechanism(s) are believed to underlie shared relations, but most critically, stating the precise ways in which the mechanism works to give rise to the relationship in question. A metaphor of a car mechanic helps to make this point. It is of use to know that car's mobility depends on its motor. This knowledge might help isolate the potential source of the problem. However, if the mechanic does not understand how the motor works, he/she has little chance of fixing a broken motor and regaining mobility. When it comes to expanding our understanding of spatial-numerical relations it is not enough to identify potential mechanisms that link spatial and numerical thought. The time is right to begin understanding why and under what specific conditions the mechanisms work, or just as importantly, fail to work. By better illuminating the learning processes that link spatial visualization and numerical competencies, we may be afforded new insights into the uniquely human ability to learn, perform, and invent abstract mathematics. This information, in turn, may prove 
critical in the assessment and design of effective mathematics curricula and intervention moving forward.

\section{References}

Alloway, T. P., \& Passolunghi, M. C. (2011). The relationship between working memory, IQ, and mathematical skills in children. Learning and Individual Differences, 21(1), 133-137.

Ackerman, P. L. (1988). Determinants of individual differences during skill acquisition: Cognitive abilities and information processing. Journal of Experimental Psychology: General, 117, 288-318.

Anderson, M. L. (2010). Neural reuse: A fundamental organizational principle of the brain. Behavioral and Brain Sciences, 33(4), 245266.

Anderson, M. L. (2016) Précis of. Behavioral and Brain Sciences 39.

Antonietti, A. (1999). Can students predict when imagery will allow them to discover the problem solution?. European Journal of Cognitive Psychology, 11(3), 407-428.

Barsalou, L. W. (2008). Grounded cognition. Annual Review of Psychology, 59, 617-645.

Barth, H. C., \& Paladino, A. M. (2011). The development of numerical estimation: Evidence against a representational shift. Developmental Science, 14(1), 125-135.

Bisiach, E., \& Luzatti, C. (1978). Unilateral neglect of representational space. Cortex, 14, 129- 133.

Boonen, A. J., van der Schoot, M., van Wesel, F., de Vries, M. H., \& Jolles, J. (2013). What underlies successful word problem solving? A path analysis in sixth grade students. Contemporary Educational Psychology, 38(3), 271-279.

Boonen, A. J., van Wesel, F., Jolles, J., \& van der Schoot, M. (2014). The role of visual representation type, spatial ability, and reading comprehension in word problem solving: An item-level analysis in elementary school children. International Journal of Educational Research, 68, 15-26.

Calabria, M., \& Rossetti, Y. (2005). Interference between number processing and line bisection: a methodology. Neuropsychologia, 43(5), 779-783.

Carpenter, P. A., \& Just, M. A. (1986). Spatial ability: An information processing approach to psychometrics. In R. J. Stenberg (Ed.), Advances in the psychology of human intelligence (Vol. 3, pp. 221-252). Hillsdale, NJ: Erlbaum.

Cheng, Y. L., \& Mix, K. S. (2014). Spatial training improves children's mathematics ability. Journal of Cognition and Development, 15(1), 2-11.

Cheung, C. N., Sung, J. Y., \& Lourenco, S. F. (2019). Does training mental rotation transfer to gains in mathematical competence? Assessment of an at-home visuospatial intervention. Psychological Research, 1-18.

Chu, M., \& Kita, S. (2011). The nature of gestures' beneficial role in spatial problem solving. Journal of Experimental Psychology: General, 140(1), 102.

Cipora, K., Hohol, M., Nuerk, H. C., Willmes, K., Brożek, B., Kucharzyk, B., \& Nęcka, E. (2016). Professional mathematicians differ from controls in their spatial-numerical associations. Psychological Research, 80(4), 710-726.

Cipora, K., Patro, K., \& Nuerk, H. C. (2015). Are spatial-numerical associations a cornerstone for arithmetic learning? The lack of genuine correlations suggests no. Mind, Brain, and Education, 9(4), 190-206.

Cornu, V., Schiltz, C., Pazouki, T., \& Martin, R. (2017). Training early visuo-spatial abilities: A controlled classroom-based intervention study. Applied Developmental Science, 1-21.
Davis, B, the Spatial Reasoning Study Group (2015). Spatial reasoning in the early years: Principles, assertions, and speculations. New York, NY: Routledge.

Dehaene, S. (2011). The number sense: How the mind creates mathematics. New York, NY: Oxford University Press.

Dehaene, S., Bossini, S., \& Giraux, P. (1993). The mental representation of parity and number magnitude. Journal of Experimental Psychology: General, 122(3), 371-396.

Dehaene, S., \& Cohen, L. (2007). Cultural recycling of cortical maps. Neuron, 56(2), 384-398.

Dehaene, S., Piazza, M., Pinel, P., \& Cohen, L. (2003). Three parietal circuits for number processing. Cognitive Neuropsychology, 20(36), 487-506.

Delgado, A. R., \& Prieto, G. (2004). Cognitive mediators and sex-related differences in mathematics. Intelligence, 32(1), 25-32.

DeStefano, D., \& LeFevre, J. A. (2004). The role of working memory in mental arithmetic. European Journal of Cognitive Psychology, 16(3), 353-386.

Fischer, M. H., \& Fias, M. H. (2005). Spatial representation of numbers. Handbook of Mathematical Cognition, 43-54.

Fischer, M. H., Mills, R. A., \& Shaki, S. (2010). How to cook a SNARC: Number placement in text rapidly changes spatial-numerical associations. Brain and cognition, 72(3), 333-336.

Fischer, U., Moeller, K., Bientzle, M., Cress, U., \& Nuerk, H. C. (2011). Sensori-motor spatial training of number magnitude representation. Psychonomic Bulletin \& Review, 18(1), 177-183.

Font, V., Godino, J. D., \& Gallardo, J. (2013). The emergence of objects from mathematical practices. Educational Studies in Mathematics, 82(1), 97-124.

Galton, F. (1880). Visualised numerals. Nature, 21(533), 252-256.

Galton, F. (1881). Visualised numerals. The Journal of the Anthropological Institute of Great Britain and Ireland, 10, 85-102.

Gerstmann, J. (1940). Syndrome of finger agnosia, disorientation for right and left, agraphia and acalculia: local diagnostic value. Archives of Neurology \& Psychiatry, 44(2), 398-408.

Gevers, W., Reynvoet, B., \& Fias, W. (2003). The mental representation of ordinal sequences is spatially organized. Cognition, 87(3), B87B95.

Gevers, W., Reynvoet, B., \& Fias, W. (2004). The mental representation of ordinal sequences is spatially organised: Evidence from days of the week. Cortex, 40, 171-172.

Giaquinto, M. (2008). Visualizing in mathematics. The Philosophy of Mathematical Practice. New York, NY: Oxford University Press.

Greeno, J. G. (1991). Number sense as situated knowing in a conceptual domain. Journal for Research in Mathematics Education, 22(3), 170-218.

Gunderson, E. A., Ramirez, G., Beilock, S. L., \& Levine, S. C. (2012). The relation between spatial skill and early number knowledge: the role of the linear number line. Developmental Psychology, 48(5), $1229-1241$.

Hadamard, J. (1945). The psychology of invention in the mathematical field. Princeton, NJ: Princeton University Press

Halpern, D. F., Benbow, C. P., Geary, D. C., Gur, R. C., Hyde, J. S., \& Gernsbacher, M. A. (2007). The science of sex differences in science and mathematics. Psychological Science in the Public Interest, 8(1), $1-51$.

Hawes, Z., Moss, J., Caswell, B., \& Poliszczuk, D. (2015). Effects of mental rotation training on children's spatial and mathematics performance: A randomized controlled study. Trends in Neuroscience and Education, 4(3), 60-68.

Hawes, Z., Moss, J., Caswell, B., Naqvi, S., \& MacKinnon, S. (2017). Enhancing children's spatial and numerical skills through a dynamic spatial approach to early geometry instruction: Effects of a 32-week intervention. Cognition and Instruction, 35(3), 236-264.

Hawes, Z., Moss, J., Caswell, B., Seo, J., \& Ansari, D. (2019a). Relations between numerical, spatial, and executive function skills and 
mathematics achievement: A latent-variable approach. Cognitive Psychology, 109, 68-90.

Hawes, Z., Sokolowski, H. M., Ononye, C. B., \& Ansari, D. (2019b). Neural underpinnings of numerical and spatial cognition: An fMRI meta-analysis of brain regions associated with symbolic number, arithmetic, and mental rotation. Neuroscience \& Biobehavioral Reviews, 103, 316-336.

Hegarty, M., \& Kozhevnikov, M. (1999). Types of visual-spatial representations and mathematical problem solving. Journal of Educational Psychology, 91(4), 684-689

Hegarty, M., \& Waller, D. (2005). Individual differences in spatial abilities. The Cambridge Handbook of Visuospatial Thinking, 121-169.

Holmes, G. (1918). Disturbances of visual orientation. The British Journal of Ophthalmology, 2(9), 449-468.

Hubbard, E. M., M. Piazza, P. Pinel, \& Dehaene (2009). Numerical and spatial intuitions: a role for posterior parietal cortex? In L. Tommasi, L. Nadel, and M. A. Peterson (Eds.), Cognitive biology: Evolutionary and developmental perspectives on mind, brain and behavior (pp. 221-246). Cambridge, MA: MIT Press

Hutchison, J. E., Lyons, I. M., \& Ansari, D. (2019). More similar than different: Gender differences in children's basic numerical skills are the exception not the rule. Child Development, 90(1), e66-e79.

Huttenlocher, J., Jordan, N. C., \& Levine, S. C. (1994). A mental model for early arithmetic. Journal of Experimental Psychology: General, 123(3), 284-296.

Johnson-Frey, S. H. (2004). The neural bases of complex tool use in humans. Trends in Cognitive Sciences, 8(2), 71-78.

Jordan, K., Heinze, H. J., Lutz, K., Kanowski, M., \& Jäncke, L. (2001). Cortical activations during the mental rotation of different visual objects. NeuroImage, 13(1), 143-152.

Just, M. A., \& Carpenter, P. A. (1985). Cognitive coordinate systems: accounts of mental rotation and individual differences in spatial ability. Psychological Review, 92(2), 137.

Kadosh, R. C., Lammertyn, J., \& Izard, V. (2008). Are numbers special? An overview of chronometric, neuroimaging, developmental and comparative studies of magnitude representation. Progress in Neurobiology, 84(2), 132-147.

Kaufman, S. B. (2007). Sex differences in mental rotation and spatial visualization ability: Can they be accounted for by differences in working memory capacity? Intelligence, 35, 211-223.

Kell, H. J., Lubinski, D., Benbow, C. P., \& Steiger, J. H. (2013). Creativity and technical innovation: Spatial ability's unique role. Psychological Science, 24(9), 1831-1836.

Kersey, A. J., Braham, E. J., Csumitta, K. D., Libertus, M. E., \& Cantlon, J. F. (2018). No intrinsic gender differences in children's earliest numerical abilities. NPJ Science of Learning, 3(1), 12.

King, M. J., Katz, D. P., Thompson, L. A., \& Macnamara, B. N. (2019). Genetic and environmental influences on spatial reasoning: A metaanalysis of twin studies. Intelligence, 73, 65-77.

Knops, A., Viarouge, A., \& Dehaene, S. (2009). Dynamic representations underlying symbolic and nonsymbolic calculation: Evidence from the operational momentum effect. Attention, Perception, \& Psychophysics, 71(4), 803-821.

Kyllonen, P. C., \& Christal, R. E. (1990). Reasoning ability is (little more than) working-memory capacity. Intelligence, 14(4), 389-433.

Kyttälä, M., Aunio, P., Lehto, J. E., Van Luit, J., \& Hautamaki, J. (2003). Visuospatial working memory and early numeracy. Educational and Child Psychology, 20, 65-76.

Kyttälä, M., \& Lehto, J. E. (2008). Some factors underlying mathematical performance: The role of visuospatial working memory and nonverbal intelligence. European Journal of Psychology of Education, 23(1), 77-94.

Lakoff, G., \& Núñez, R. E. (2000). Where mathematics comes from: How the embodied mind brings mathematics into being. New York, NY: Basic Books.
LeFevre, J. A., Jimenez Lira, C., Sowinski, C., Cankaya, O., Kamawar, D., \& Skwarchuk, S. L. (2013). Charting the role of the number line in mathematical development. Frontiers in Psychology, 4, 641.

Li, Y., \& Geary, D. C. (2017). Children's visuospatial memory predicts mathematics achievement through early adolescence. PLoS One, 12(2), e0172046.

Link, T., Moeller, K., Huber, S., Fischer, U., \& Nuerk, H. C. (2013). Walk the number line-An embodied training of numerical concepts. Trends in Neuroscience and Education, 2(2), 74-84.

Loetscher, T., Bockisch, C., Nicholls, M. E. R., \& Brugger, P. (2010). Eye position predicts what number you have in mind. Current Biology, 20, R264-R265

Lohman, D. F. (1988). Spatial abilities as traits, processes, and knowledge. In R. J. Sternberg (Ed.), Advances in the psychology of human intelligence (Vol. 4, pp. 181-248). Hillsdale, NJL Erlbaum.

Lohman, D. F. (1996). Spatial ability and G. In I. Dennis \& P. Tapsfield (Eds.), Human abilities: Their nature and assessment (pp. 97-116). Hillsdale, NJ: Lawrence Erlbaum.

Lourenco, S. F., Cheung, C. N., \& Aulet, L. S. (2018). Is visuospatial reasoning related to early mathematical development? A critical review. In Heterogeneity of Function in Numerical Cognition (pp. 177-210). London, UK: Academic Press.

Lowrie, T., Logan, T., \& Ramful, A. (2017). Visuospatial training improves elementary students' mathematics performance. British Journal of Educational Psychology, 87(2), 170-186.

Marghetis, T., Núñez, R., \& Bergen, B. K. (2014). Doing arithmetic by hand: Hand movements during exact arithmetic reveal systematic, dynamic spatial processing. The Quarterly Journal of Experimental Psychology, 67(8), 1579-1596.

Mathieu, R., Epinat-Duclos, J., Sigovan, M., Breton, A., Cheylus, A., Fayol, M., ... Prado, J. (2017). What's Behind a "+" Sign? Perceiving an Arithmetic Operator Recruits Brain Circuits for Spatial Orienting. Cerebral Cortex, 28(5), 1673-1684.

Mayer, E., Martory, M. D., Pegna, A. J., Landis, T., Delavelle, J., \& Annoni, J. M. (1999). A pure case of Gerstmann syndrome with a subangular lesion. Brain, 122(6), 1107-1120.

McCrink, K., Dehaene, S., \& Dehaene-Lambertz, G. (2007). Moving along the number line: Operational momentum in nonsymbolic arithmetic. Perception \& Psychophysics, 69(8), 1324-1333.

Mix, K. S., \& Cheng, Y. L. (2012). The relation between space and math: developmental and educational implications. Advances in Child Development and Behavior, 42, 197-243.

Mix, K. S., Levine, S. C., Cheng, Y. L., Young, C., Hambrick, D. Z., Ping, R., \& Konstantopoulos, S. (2016). Separate but correlated: The latent structure of space and mathematics across development. Journal of Experimental Psychology: General, 145(9), 1206-1227.

Miyake, A., Friedman, N. P., Rettinger, D. A., Shah, P., \& Hegarty, M. (2001). How are visuospatial working memory, executive functioning, and spatial abilities related? A latent-variable analysis. Journal of Experimental Psychology: General, 130(4), 621-640.

Moss, J., Bruce, C. D., Caswell, B., Flynn, T., \& Hawes, Z. (2016). Taking shape: Activities to develop geometric and spatial thinking. Grades K-2. Pearson Canada Incorporated.

Newcombe, N. S. (2010). Picture this: Increasing math and science learning by improving spatial thinking. American Educator, 34(2), 29-35.

Newcombe, N. S., Levine, S. C., \& Mix, K. S. (2015). Thinking about quantity: The intertwined development of spatial and numerical cognition. Wiley Interdisciplinary Reviews: Cognitive Science, 6(6), 491-505.

Paivio, A. (1983). The mind's eye in arts and science. Poetics, 12(1), 1-18.

Paivio, A. (2013). Imagery and verbal processes. New York, NY: Psychology Press.

Patro, K., Fischer, U., Nuerk, H. C., \& Cress, U. (2016). How to rapidly construct a spatial-numerical representation in preliterate children (at least temporarily). Developmental Science, 19(1), 126-144. 
Ramani, G. B., \& Siegler, R. S. (2008). Promoting broad and stable improvements in low-income children's numerical knowledge through playing number board games. Child Development, 79(2), 375-394.

Redick, T. S., Shipstead, Z., Wiemers, E. A., Melby-Lervåg, M., \& Hulme, C. (2015). What's working in working memory training? An educational perspective. Educational Psychology Review, 27(4), 617-633.

Root-Bernstein, R. S. (1985). Visual thinking: The art of imagining reality. Transactions of the American Philosophical Society, 75(6), 50-67.

Schneider, M., Beeres, K., Coban, L., Merz, S., Susan Schmidt, S., Stricker, J., \& De Smedt, B. (2017). Associations of non-symbolic and symbolic numerical magnitude processing with mathematical competence: A meta-analysis. Developmental Science, 20(3), e12372.

Schneider, M., Merz, S., Stricker, J., De Smedt, B., Torbeyns, J., Verschaffel, L., \& Luwel, K. (2018). Associations of number line estimation with mathematical competence: A meta-analysis. Child Development, 89(5), 1467-1485.

Seydell-Greenwald, A., Ferrara, K., Chambers, C. E., Newport, E. L., \& Landau, B. (2017). Bilateral parietal activations for complex visualspatial functions: Evidence from a visual-spatial construction task. Neuropsychologia, 106, 194-206.

Shaki, S., Fischer, M. H., \& Petrusic, W. M. (2009). Reading habits for both words and numbers contribute to the SNARC effect. Psychonomic Bulletin \& Review, 16(2), 328-331.

Siegler, R. S., \& Ramani, G. B. (2009). Playing linear number board games - but not circular ones - improves low-income preschoolers' numerical understanding. Journal of Educational Psychology, 101(3), 545-560.

Simms, V., Clayton, S., Cragg, L., Gilmore, C., \& Johnson, S. (2016). Explaining the relationship between number line estimation and mathematical achievement: the role of visuomotor integration and visuospatial skills. Journal of Experimental Child Psychology, 145, 22-33.

Sokolowski, H. M., Fias, W., Mousa, A., \& Ansari, D. (2017). Common and distinct brain regions in both parietal and frontal cortex support symbolic and nonsymbolic number processing in humans: A functional neuroimaging meta-analysis. Neuroimage, 146, 376-394.

Stengel, E. (1944). Loss of spatial orientation, constructional apraxia and Gerstmann's syndrome. Journal of Mental Science, 90(380), 753760.

Tam, Y. P., Wong, T. T. Y., \& Chan, W. W. L. (2019). The relation between spatial skills and mathematical abilities: The mediating role of mental number line representation. Contemporary Educational Psychology, 56, 14-24.
Titze, C., Jansen, P., \& Heil, M. (2010). Mental rotation performance and the effect of gender in fourth graders and adults. European Journal of Developmental Psychology, 7(4), 432-444.

Tolar, T. D., Lederberg, A. R., \& Fletcher, J. M. (2009). A structural model of algebra achievement: computational fluency and spatial visualisation as mediators of the effect of working memory on algebra achievement. Educational Psychology, 29(2), 239-266.

Toomarian, E. Y., \& Hubbard, E. M. (2018). On the genesis of spatialnumerical associations: Evolutionary and cultural factors coconstruct the mental number line. Neuroscience \& Biobehavioral Reviews.

Tosto, M. G., Hanscombe, K. B., Haworth, C. M., Davis, O. S., Petrill, S. A., Dale, P. S., ... Kovas, Y. (2014). Why do spatial abilities predict mathematical performance?. Developmental Science, 17(3), 462470.

Uttal, D. H., \& Cohen, C. A. (2012). Spatial thinking and STEM education: When, why and how. Psychology of Learning and Motivation, 57, 147-181.

Uttal, D. H., Meadow, N. G., Tipton, E., Hand, L. L., Alden, A. R., Warren, C., \& Newcombe, N. S. (2013). The malleability of spatial skills: A meta-analysis of training studies. Psychological Bulletin, 139(2), 352.

van Dijck, J. P., \& Fias, W. (2011). A working memory account for spatial-numerical associations. Cognition, 119(1), 114-119.

Viarouge, A., Hubbard, E. M., \& McCandliss, B. D. (2014). The cognitive mechanisms of the SNARC effect: An individual differences approach. PloS one, 9(4), e95756.

Wai, J., Lubinski, D., \& Benbow, C. P. (2009). Spatial ability for STEM domains: Aligning over fifty years of cumulative psychological knowledge solidifies its importance. Journal of Educational Psychology, 101, 817-835.

Walsh, V. (2003). A theory of magnitude: common cortical metrics of time, space and quantity. Trends in Cognitive Sciences, 7(11), 483488.

Wei, W., Yuan, H., Chen, C., \& Zhou, X. (2012). Cognitive correlates of performance in advanced mathematics. British Journal of Educational Psychology, 82(1), 157-181.

Wright, R., Thompson, W. L., Ganis, G., Newcombe, N. S., \& Kosslyn, S. M. (2008). Training generalized spatial skills. Psychonomic Bulletin \& Review, 15(4), 763-771.

Zacks, J. M. (2008). Neuroimaging studies of mental rotation: a metaanalysis and review. Journal of Cognitive Neuroscience, 20(1), 119.

Zorzi, M., Priftis, K., \& Umiltà, C. (2002). Brain damage: neglect disrupts the mental number line. Nature, 417(6885), 138-139.

Publisher's note Springer Nature remains neutral with regard to jurisdictional claims in published maps and institutional affiliations. 